\title{
ESTIGMA E TESTE RÁPIDO NAATENÇÃO BÁSICA: PERCEPÇÃO DE USUÁRIOS E PROFISSIONAIS
}

\section{Stigma and rapid testing in Primary Care: users' and professionals' perception \\ Estigma y prueba rápida de la atención básica: percepción de usuarios y profesionales}

\author{
Raquel de Andrade Souza Ew (OrcID) \\ Pontifícia Universidade Católica do Rio Grande do Sul - PUCRS - Porto Alegre (RS) - Brasil \\ Guilherme Severo Ferreira (Lattes) \\ Pontifícia Universidade Católica do Rio Grande do Sul - PUCRS - Porto Alegre (RS) - Brasil \\ Larissa Moraes Moro (OrcID) \\ Pontifícia Universidade Católica do Rio Grande do Sul - PUCRS - Porto Alegre (RS) - Brasil
}

Kátia Bones Rocha (OrcID)

Pontifícia Universidade Católica do Rio Grande do Sul - PUCRS - Porto Alegre (RS) - Brasil

\section{RESUMO}

Objetivo: Analisar como o estigma e a discriminação estão presentes no cotidiano de usuários e profissionais no contexto de implementação do teste rápido para HIVIAIDS na Atenção Básica. Métodos: Estudo qualitativo, de caráter exploratório, realizado em 2015, em 15 unidades de saúde de Porto Alegre, Brasil. Participaram 64 pessoas, sendo 34 usuários e 30 profissionais de saúde com diferentes formações. Os dados foram coletados através de entrevistas semiestruturadas e organizados a partir da análise temática das falas dos participantes. Resultados: O estigma associado ao HIVIAIDS se faz presente nas diferentes falas dos entrevistados. Os participantes apresentaram semelhanças na indicação de quem deveria realizar o teste rápido de HIV/ AIDS, citando usuários de drogas, adolescentes e mulheres. Os entrevistados referem estigma e discriminação na comunidade e nos serviços de saúde, sendo que os profissionais afirmam que existe preconceito por parte de membros da equipe e dos próprios usuários do serviço, sem implicarem-se nesse processo. Considerar a AIDS como uma doença qualquer é uma forma de neutralizar a identificação do estigma, não reconhecendo as especificidades históricas do HIV e as singularidades das pessoas afetadas. Conclusão: $O$ desafio da Atenção Básica é reconhecer quais são os conceitos que embasam a identificação de pessoas vulneráveis ao HIVIAIDS, para que não se reproduzam estigmas que entravam a promoção de saúde.

Descritores: Estigma Social; Atenção Primária à Saúde; Sorodiagnóstico da AIDS.

\begin{abstract}
Objective: To analyze how stigma and discrimination related to Human Immunodeficiency Virus/Acquired Immunodeficiency Syndrome (HIVIAIDS) are presented in the everyday lives of users and professionals in the context of the implantation of rapid testing for HIV and other Sexually Transmitted Infections (STI) in Primary Health Care. Methods: Qualitative, exploratory study, performed in 2015 in 15 health care units of Porto Alegre, Brazil. Participants were 64 people, 34 users and 30 health professionals of different fields. Data was collected using semi-structured interview and organized based on the thematic analysis of the participants'statements. Results: The stigma associated with HIVIAIDS is present in the different speeches of the interviewees. Participants showed similarities in indicating who should take the rapid HIV test, citing drug users, adolescents and women. The respondents perceive stigma and discrimination in the community and health services, and the professionals say that there is prejudice on the part of staff members and even the service users, not becoming involved in this process. Considering Aids as any disease is a way of neutralizing the identification of stigma, which does not recognize the historical specificities of HIV and the singularities of the people affected. Conclusion: The challenge faced by Primary Health Care is to recognize the concepts that underpin the identification of individuals vulnerable to HIVIAIDS, so as to avoid reproducing stigma that hamper health promotion .
\end{abstract}

Descriptors: Stigma; Primary Health Care; Aids Serodiagnosis. 


\section{RESUMEN}

Objetivo: Analizar como el estigma y la discriminación están presentes en el cotidiano de los usuarios y profesionales en el contexto de la implementación de la prueba rápida del VIH/SIDA en la Atención Básica. Métodos: Estudio cualitativo de carácter exploratorio realizado en 2015 en 15 unidades de salud de Porto Alegre, Brasil. Participaron 64 personas y de esas, 34 usuarios y 30 profesionales sanitarios con distintas formaciones. Los datos fueron recogidos de las entrevistas semiestructuradas y organizados a partir del análisis temático de las hablas de los participantes. Resultados: El estigma asociado al VIH/SIDA está presente en las distintas hablas de los entrevistados. Los participantes presentaron similitudes para la indicación de quien debería realizar la prueba rápida deI VIH/SIDA citando los usuarios de drogas, los adolescentes y las mujeres. Los entrevistados refieren el estigma y la discriminación de la comunidad y los servicios de salud y los profesionales afirman que hay prejuicio de parte de los miembros del equipo y de los propios usuarios del servicio sin implicarse en ese proceso. Considerar la SIDA como una enfermedad cualquiera es una manera de neutralizar la identificación del estigma sin reconocer las especificidades históricas del VIH y las singularidades de las personas afectadas. Conclusión: El desafío de la Atención Básica es reconocer cuales son los conceptos que basan la identificación de las personas vulnerables al VIH/SIDA para que no se reproduzcan estigmas que entraban la promoción de la salud.

Descriptores: Estigma Social; Atención Primaria de Salud; Sorodiagnóstico del SIDA.

\section{INTRODUÇÃO}

As taxas de epidemia de Vírus da Imunodeficiência Humana/Síndrome da Imunodeficiência Adquirida (HIV/ AIDS) seguem sendo uma preocupação global. Atualmente, 36,7 milhões de pessoas vivem com HIV no mundo (1.). A incidência em 2016 de AIDS apresenta taxas, para cada cem mil habitantes, de 19,1 no Brasil; sendo especificamente de 34,7 no Rio Grande do Sul e 74 em Porto Alegre ${ }^{(2)}$.

O estigma associado ao HIVIAIDS está presente no cotidiano das pessoas que vivem com o vírus, refletindo no acesso à saúde, nas oportunidades de trabalho e na integração na comunidade ${ }^{(1)}$. O estigma se configura como a restrição da imagem das pessoas às suas características não desejadas em determinado contexto, que apontam alguma falta, defeito ou desvantagem ${ }^{(3)}$. Estigma é definido como um processo social ligado às relações de poder $\mathrm{e}$ dominação que derivam em estereótipos, categorizações e desprezo social, de forma que, de modo discriminatório, grupos são valorizados e outros, desvalorizados ${ }^{(4,5)}$.

A discriminação associada à AIDS emana do estigma relacionado ao histórico da doença. A infecção do HIV permanece associada a julgamentos morais relacionados ao sexo e sexualidade, uma vez que se caracteriza, predominantemente, pela transmissão sexual ${ }^{(1)}$. As pesquisas sobre estigma relacionadas ao HIV e à AIDS tendem a restringir-se à descrição das experiências e a percepções individuais. Dessa maneira, os estudos não consideram o estigma como um processo social interconectado com outros eixos, como gênero, pobreza, sexualidade e raça/ etnia, que produzem desigualdades ${ }^{(6)}$.

De forma geral, conceitua-se estigma e discriminação como fenômenos produzidos por dinâmicas de poder, dominação e coerção social, que reforçam a exclusão de certas pessoas, sujeitando-as a menor qualidade de condições de saúde ${ }^{(4,7)}$. Pesquisas discutem a relação da homofobia com a produção de estigma associado ao HIV e à AIDS, especialmente em contextos nos quais algumas características são comuns em face à diversidade sexual, tais como: condições materiais de vida instáveis, normas tradicionais de gênero e situações de violência( ${ }^{(8-10)}$.

Cabe destacar que, no contexto da saúde coletiva, a partir dos anos 2000, o Ministério da Saúde tem desenvolvido diretrizes para o cuidado em HIVIAIDS na Atenção Básica (AB), como a inserção do aconselhamento e o incentivo para a oferta diagnóstica do HIV ${ }^{(11)}$. Em 2012, iniciou-se a implantação da descentralização do teste rápido de HIV para o contexto da $A B^{(12)}$.

O teste rápido é uma tecnologia que visa atender às necessidades relacionadas à prevenção de HIVIAIDS e à promoção da saúde ${ }^{(13)}$. A Política Nacional de Promoção da Saúde (PNPS) tem como um dos propósitos a implementação de ações dirigidas à AB. Essa política possibilita a visibilidade dos fatores que colocam a saúde da população em risco, considerando os contextos populacionais, visando à redução de vulnerabilidades ${ }^{(14 .)}$. Nesse sentido, o teste rápido se enquadra nessa perspectiva, visto que proporciona, além da oferta diagnóstica, o aconselhamento, que consiste na troca de informação, suporte emocional e gestão de risco ${ }^{(12)}$.

É discutido pela literatura o quanto a política de territorialização dos usuários do Sistema Único de Saúde (SUS) pode ser um elemento facilitador do acesso à saúde, tendo em vista os atravessamentos de estigma e discriminação que perpassam o atendimento às pessoas com HIVIAIDS ${ }^{(15)}$. Um estudo identificou que os usuários da $A B$ se sentiam 
preocupados quanto ao sigilo do diagnóstico de HIVIAIDS, com receio de serem percebidos na comunidade devido ao preconceito social, o que não ocorria nas demais situações de atendimento ${ }^{(16)}$.

A descentralização do teste rápido de HIV e outras Infecções Sexualmente Transmissíveis (IST) para a AB traz uma mudança significativa no atendimento, antes realizado prioritariamente em Centros Especializados de Testagens e Aconselhamento (CTA) e, atualmente, disponível nas unidades de referência próximo à moradia dos usuários. A literatura aponta que as crenças estigmatizantes dos profissionais podem se tornar uma barreira no acesso à saúde ${ }^{(17,18)}$.

Nesse sentido, este estudo tem como conjetura: como a política de descentralização do teste rápido para HIV/ AIDS na $A B$ impacta a relação entre usuários e profissionais?

Dessa forma, o objetivo deste estudo é analisar como o estigma e a discriminação estão presentes no cotidiano de usuários e profissionais no contexto de implementação do teste rápido para HIVIAIDS na Atenção Básica.

\section{MÉTODOS}

Trata-se de um estudo qualitativo, exploratório e descritivo(19), realizado no ano de 2015, em 15 unidades de saúde da Atenção Básica (AB) de Porto Alegre, Rio Grande do Sul, Brasil, sendo oito Unidades Básicas de Saúde (UBS) e sete de Estratégia de Saúde da Família (ESF). A escolha das unidades de saúde ocorreu por sorteio(19) entre os oito serviços que mais realizavam o teste rápido e os oito serviços que menos realizavam, sendo que uma unidade de saúde não aceitou participar da pesquisa. Esses dados foram obtidos em parceria com a Coordenação de HIV da Secretaria Municipal de Saúde, a partir de levantamento interno não disponibilizado publicamente.

Participaram 64 pessoas, escolhidas por conveniência ${ }^{(19)}$, sendo 34 usuários e 30 profissionais da $A B$ com diferentes formações: dezenove enfermeiros, três técnicos de enfermagem, dois agentes comunitários de saúde, dois médicos ginecologistas, um médico da família, dois cirurgiões-dentistas e um nutricionista. Os coordenadores dos serviços foram contatados via telefone para apresentação da pesquisa e agendamento das visitas. Os usuários e profissionais foram convidados para participar do estudo nas suas unidades de referência. Tomaram parte apenas os profissionais que haviam recebido a capacitação para realização do teste rápido e usuários maiores de 18 anos. O número da amostra foi determinado na concepção da pesquisa, considerando a participação de, pelo menos, dois profissionais e dois usuários por unidade de saúde. Não foram incluídos aqueles participantes que não atingiram as recomendações anteriormente citadas.

Para a coleta de dados, utilizaram-se entrevistas semiestruturadas, com questões referentes à implantação do teste rápido na $A B$, partindo da análise de três perguntas disparadoras: 1) "Na sua opinião, quem deve realizar o teste rápido para HIV?"; 2) "Você percebe a existência de estigma/discriminação no serviço de saúde e/ou na comunidade?"; e 3) Para profissionais: "Você se testaria na sua unidade de saúde?"; e, para usuários: "Você faria tratamento para detecção do HIV na sua unidade de saúde?".

As entrevistas duraram aproximadamente 30 minutos, e foram gravadas e transcritas na íntegra. Para a análise dos dados, utilizou-se a análise temática, conforme os seguintes passos: transcrição detalhada das entrevistas; codificação inicial dos temas mais relevantes a partir da exploração do material; escolha e definição dos temas relacionados; releitura e recodificação das entrevistas; organização e interpretação dos dados; redação da análise de dados interligando narrativa analítica e extratos ilustrativos ${ }^{(20)}$. Para a sistematização dos relatos dos entrevistados, utilizaram-se os seguintes eixos temáticos: Indicação de quem deveria realizar o teste: o atravessamento do estigma nas diferentes populações; Testagem e tratamento na Atenção Básica: fatores interferentes na decisão; e Identificação de estigma e discriminação no serviço e na comunidade.

Este estudo faz parte de uma pesquisa maior intitulada "Avaliação do processo de implantação da política de descentralização do teste rápido de HIV na AB a partir da percepção de matriciadores, profissionais e usuários". As questões éticas foram asseguradas, sendo a pesquisa submetida e aprovada pelos Comitês de Ética em Pesquisa da Pontifícia Universidade Católica do Rio Grande do Sul e da Secretaria Municipal de Saúde de Porto Alegre (Parecer $n^{\circ}$ 981.527). Todos os participantes assinaram o Termo de Consentimento Livre e Esclarecido. Para preservar a identidade dos participantes, identificaram-se as falas com "P" para profissionais e " $U$ " para usuários, sem mencionar as gerências e os serviços de origem.

\section{RESULTADOS E DISCUSSÃO}

Neste espaço serão apresentados os eixos temáticos que emergiram após a análise das falas. Elaborou-se uma síntese interpretativa em que as falas dos participantes dialogam com o cenário do estigma do HIVIAIDS e o 
contexto do teste rápido na $\mathrm{AB}$. Na sequência, apresentam-se os três eixos temáticos construídos: Indicação de quem deveria realizar o teste: o atravessamento do estigma nas diferentes populações; Testagem e tratamento na atenção básica: fatores interferentes na decisão; e Identificação de estigma e discriminação no serviço e na comunidade.

\section{Indicação de quem deveria realizar o teste: o atravessamento do estigma nas diferentes populações}

Neste eixo, apresenta-se a indicação de quem deveria realizar o teste rápido. Ao serem questionados, os entrevistados recorreram a respostas generalistas, indicando com maior frequência que todas as pessoas estão sujeitas a infecção pelo vírus. Os participantes referiram não existir mais um grupo de risco e que ter relações sexuais já seria um indicativo para exposição ao vírus, como pode ser observado:

"Acho difícil pensar num grupo mais vulnerável. Antes diziam que eram os "gays", mas isso já passou faz muito tempo. Acho que não tem um grupo mais vulnerável. Acho que todo mundo." (U8)

Percebe-se que a fala articula a ideia de que todas as pessoas precisam ter cuidado com o risco de adquirir o HIV. Isto pode estar associado à mobilização da sociedade civil com a gestão pública diante da epidemia do HIV/ AIDS, ao buscar estratégias para reduzir a vulnerabilidade das populações atingidas no início da epidemia ${ }^{(21)}$. A partir das políticas de enfrentamento ao HIVIAIDS no Brasil, retirou-se, paulatinamente, o foco sobre populações específicas, reconhecendo que o risco de infecção está vinculado às práticas sexuais sem uso de preservativos.

As respostas generalizadas dos participantes podem ser uma forma automática de não caracterizar uma visão estigmatizante. Por outro lado, nota-se que os entrevistados identificam diferentes grupos para realizar o teste prioritariamente, como usuários de drogas, mulheres e adolescentes/jovens. Dentre eles, destacam-se os pontos associados com a vulnerabilidade de usuários de drogas, que dizem respeito à situação de pobreza, à falta de procura por atendimento e ao compartilhamento de seringas:

"Aqui, no bairro, tem muitos drogados, pessoas com pouco estudo, que se tornam ignorantes." (U19)

"Eu gostaria de testar o pessoal que a gente chama de 'Carandiru' ali, que é um cantinho da vila, que é o pessoal da drogadição." (P26)

Percebe-se que os participantes identificam a intersecção entre estigma e pobreza. O estigma está diretamente relacionado aos eixos de desigualdade social que operam nas interações sociais ${ }^{(22)}$ entre prostitutas, mulheres que abortam e mulheres vivendo com o vírus da imunodeficiência humana (HIV. Sendo assim, as desigualdades sociais potencializam a produção de estigmas. Essas desigualdades se estendem para diferentes populações, como adolescentes, mulheres, homossexuais e idosos. A discriminação geracional, o sexismo, o racismo e a homofobia estão presentes, tanto de forma velada como explícita, na sociedade brasileira ${ }^{(23)}$.

Em relação aos adolescentes, os profissionais elencaram como motivos para o aumento da incidência das taxas de HIV nesse grupo o fato de não usarem preservativo. Conforme os usuários, os jovens devem ser testados por estarem mais suscetíveis ao uso de drogas e à gravidez não planejada. A literatura aponta que o déficit de informações associadas às questões socioculturais e a precária orientação preventiva relacionada ao HIV têm influenciado na prática sexual desprotegida, incrementando os índices de HIV ${ }^{(24)}$.

As mulheres foram identificadas com maior vulnerabilidade ao HIV em diferentes fases e situações da vida. Isto pode ser observado nos seguintes trechos:

"Vestimenta, não é? Na escola, a gente já foi algumas vezes e tu vê (sic) como as gurias se comportam: tudo de namorado, menor... E até pelas gestantes aqui que tiveram filhos com treze anos. Acredito que essas sejam as mais suscetíveis." (P5)

"Tem muita adolescente grávida, e gravidez quer dizer que não usou o preservativo, então, pode passar (vírus). Teria que focar mais na cabeça das adolescentes." (U21)

"Mulheres casadas, é o público mais vulnerável, que acha que está protegido e não está." (P1)

Observa-se que, para os participantes, ser mulher indica maior susceptibilidade ao risco de infecção, mostrando um importante atravessamento de preconceito relacionado ao gênero. Em contraposição, há uma tendência de redução da incidência de casos de infecção entre as mulheres, tanto no Brasil( ${ }^{(2)}$ como no Rio Grande do Sul(25).

$\mathrm{Na}$ atual pesquisa, é possível perceber nas falas dos entrevistados o quanto a sexualidade feminina é perpassada pela percepção de risco que envolve as diversas manifestações e momentos de vida. Por aparecer tanto na fala dos usuários quanto dos profissionais, esse aspecto reflete como essas concepções são estigmatizantes e presentes 
culturalmente. Assim, o desenvolvimento do corpo, a gestação e a vida sexual das mulheres sempre foram motivo de cuidado e controle.

Um estudo avaliou que as representações sociais sobre a exposição da mulher ao HIVIAIDS, quando associadas negativamente, ou seja, quando as culpabilizam pela infecção, comprometem a qualidade da assistência à saúde e o vínculo entre profissional de saúde e usuária ${ }^{(26)}$. Outra pesquisa aponta que os serviços de saúde nem sempre viabilizam meios das mulheres exercerem suas sexualidades com poucos riscos. Isto faz com que ocorra, por exemplo, a desvinculação entre oferta de contracepção e prevenção do HIV, e falhas no aconselhamento pré e pós-testagem, especialmente na não abordagem de práticas sexuais e gestão de risco(27).

Os entrevistados não apontaram os homossexuais como público-alvo para testagem. Os profissionais observam que houve uma mudança no perfil dos infectados por HIVIAIDS, considerando uma maior generalização da epidemia:

"Se é um adolescente, nem sabe de 1980, de quem eram as pessoas que adoeciam. Lembro dessa história: 'se tu não és homossexual, não vais pegar'. E o que aconteceu? O que está acontecendo agora: idoso, adolescente, relações estáveis. Acho que o que mais dificulta é o preconceito do HIV." (P20)

Percebe-se que a questão cultural da associação direta entre a população homossexual e o HIV pode ser um fator de risco que dificulta a autopercepção de vulnerabilidade por outras populações. Além disso, entre os profissionais da saúde, apresenta-se uma quebra do paradigma da epidemia estar vinculada à comunidade homossexual. Isto pode ser um reflexo positivo das políticas voltadas para a prevenção do HIV, que têm desconstruído essa concepção estigmatizante ao longo desses 30 anos ${ }^{(28)}$.

Os profissionais do presente estudo ainda apontaram os idosos como outro público de atenção por apresentarem vida sexual ativa e não possuírem o hábito do uso de preservativo. Destaca-se que os usuários não fazem referência ao risco de idosos contraírem o vírus, corroborando o que é apontado na literatura: na concepção social, a sexualidade de idosos é negada e tratada com preconceito(29,30).

Os estudos assinalam que há invisibilidade da vida sexual dos idosos, e os profissionais da área da saúde partilham dessa crença ${ }^{(31,32)}$. No entanto, vê-se um aumento de $20 \%$ da taxa de detecção do HIV na população idosa (mais de 60 anos) entre 2006 a $2015^{(25)}$. Assim, a visão desse profissional (P20) mostra-se sensível a essa condição de invisibilidade.

Cabe destacar que as metas globais da Organização Mundial da Saúde (OMS), através do Programa Conjunto das Nações Unidas sobre HIVIAIDS (Joint United Nations Program on HIVIAIDS - UNAIDS), visam um controle da epidemia até 2020 , com o objetivo "90-90-90", isto é, $90 \%$ das pessoas com HIV cientes do diagnóstico, $90 \%$ dos diagnosticados em tratamento e $90 \%$ das pessoas em tratamento com carga viral indetectável. Essas metas visam à promoção da saúde e consideram todas as populações e territórios, sem discriminação(1).

Nesse sentido, percebe-se que as respostas dos profissionais do atual estudo estão alinhadas com essa visão propositiva diagnóstica. No entanto, para viabilizar a promoção de saúde, é necessário perceber as singularidades de exposição conforme os atributos específicos de infecção ao HIV de diferentes populações, no intuito de promover a gestão de risco das pessoas frente suas vulnerabilidades particulares ${ }^{(14)}$.

\section{Testagem e tratamento na atenção básica: fatores interferentes na decisão}

No eixo referente à testagem e tratamento na $A B$, é apresentada uma disponibilidade dos usuários em realizar o tratamento para HIVIAIDS na unidade de seu território. Dentre os motivos apresentados, destacam-se a facilidade de acesso e a proximidade da unidade com suas residências. Além disso, alguns relataram como justificativa o vínculo estabelecido com os profissionais de saúde:

"Faria, eu sou mais acostumada com esse posto, eu não sou de ir para hospital. Me sinto bem aqui." (U1)

"Acho que eu me trataria aqui. Os outros lugares são muito longe (...). Aqui, na unidade, tu conheces todo mundo. Tu sabes com quem pode conversar mais abertamente, é mais difícil ir para um lugar estranho, que não se conhece ninguém." (U21)

Os exemplos dessas falas apresentam a descentralização da atenção e do tratamento do HIV como uma iniciativa positiva, facilitando o fortalecimento de vínculos, ao considerar que na $A B$ há uma maior proximidade entre o serviço e a comunidade ${ }^{(33,34)}$. Assim, o vínculo estabelecido entre usuários e profissionais parece funcionar como elemento protetivo contra o estigma e a discriminação, promovendo maior liberdade e abertura ao diálogo entre usuário e profissional. 
Por outro lado, outros usuários dividiram suas opiniões, elencando receio em relação ao sigilo da equipe, discriminação, apreensão de ser identificado na comunidade e a falta de confiança no tratamento oferecido:

"Pela discriminação, talvez não faria. Se eu tivesse (HIV), com certeza não, porque a discriminação é brutal." (U9) "Não, porque eu não confio muito (na equipe do posto), um hospital é outra coisa." (U13)

Percebe-se uma cisão de posicionamento conforme o vínculo estabelecido com o serviço. De um lado, os usuários que se sentem acolhidos pela equipe priorizam o atendimento na unidade do seu território. De outro, aqueles que não confiam na equipe preferem ser atendidos onde possam manter o anonimato, devido ao medo de sofrer discriminação. A literatura indica que a preferência pelo atendimento na $A B$ está diretamente relacionada com a implantação do acolhimento nos serviços ${ }^{(35)}$.

Semelhante às respostas dos usuários, os profissionais entrevistados também indicaram que o vínculo com a equipe seria um facilitador para a testagem nas unidades básicas:

"Faria, tranquilamente. Até me sentiria mais segura fazendo na minha área, com profissionais que eu tenho um certo vínculo, do que fazendo numa outra área, com um desconhecido." (P25)

Ressalta-se, ainda, que os profissionais afirmaram que realizariam o teste na sua unidade de trabalho ou na unidade de referência por terem certeza de o diagnóstico atual ser negativo. Dessa forma, isto pode ter influenciado na segurança das respostas quanto à testagem, uma vez que a ansiedade e hesitação à testagem estão relacionadas à autopercepção de exposição ao risco de infecção:

"Eu faria, porque eu sei que eu sou negativa (HIV). Se eu desconfiasse que sou positiva, eu não sei. É uma coisa difícil, vou pedir para uma colega fazer o teste rápido em mim, porque eu desconfio que eu posso estar positivo por algum motivo, e (se) dá positivo, não sei como eu trabalharia isso." (P16)

No entanto, outros profissionais apresentaram uma tendência a não realizar o teste rápido na AB. Eles elencaram como justificativa o estigma e a discriminação, demonstrando medo de rejeição dos pares em relação à possibilidade de diagnóstico positivo:

"É muito complicado. Enquanto você cuida, é uma coisa, mas quando você vai ser cuidado, já passa para outro princípio. Às vezes, a pessoa fica com muito receio, não quer se expor no ambiente de trabalho, mas, como profissional de saúde, não vejo problema em fazer. Acho que essa foi a pergunta mais difícil, me colocar no lugar de usuário." (P13)

Nota-se que a resposta dos profissionais da presente pesquisa em relação à disponibilidade ao teste e tratamento relaciona-se aos papéis sociais desenvolvidos. Na posição de profissional, eles não identificam motivos para não realizar o teste, devido aos princípios das políticas de prevenção ao avanço da epidemia. Na posição de usuários, as questões afetivas e relacionadas ao estigma afloram, sendo destacado o medo da exposição diante a reação de colegas e comunidade. Logo, percebe-se que a rigidez dos papéis sociais desempenhados dificulta o processo de vinculação entre profissionais e usuários. Dessa maneira, a promoção de empatia se destaca como elemento fundamental no processo de redução de estigma ao $\operatorname{HIVIAIDS}^{(36)}$, sendo esse um fator primordial para aproximação e estabelecimento de confiança entre profissionais e usuários, o que propicia um cenário favorável para a promoção da saúde.

\section{Identificação de estigma e discriminação no serviço e na comunidade}

Neste eixo é apresentado como os entrevistados percebem o estigma e a discriminação no serviço e na comunidade. Entre os profissionais, poucos mencionaram a presença de estigma e discriminação relacionados ao HIV, sendo a maior parte dos eventos descritos dirigidos ao preconceito do usuário. Eles relatam que os usuários manifestam receio em realizar o teste e serem reconhecidos, como também expressam dificuldade em conviver com pessoas com HIV. Na perspectiva deles, os usuários usam estratégias protetivas não vinculadas às formas de transmissão do HIV, como evitar abraços, não compartilhar utensílios domésticos e ambientes de circulação:

"Eu tenho um caso em que a família acabou isolando o irmão, fez uma casinha separada, porque ele tinha uma sobrinha que estava grávida... então, ainda existe uma discriminação bem forte." (P12)

Destaca-se que os profissionais identificam preconceito por parte de outros membros da equipe. Os entrevistados relatam que, apesar de os profissionais possuírem o conhecimento técnico sobre as maneiras de transmissão do 
HIV, alguns apresentam receio de infecção pelo contato com os usuários. Com isso, salienta-se que, apesar das campanhas governamentais para o esclarecimento do HIVIAIDS, o conhecimento não é suficiente para transformar as condutas em relação às pessoas com o vírus e às práticas de prevenção ${ }^{(37)}$.

Da mesma forma, ao serem questionados sobre a existência de estigma e discriminação, poucos usuários também indicaram a presença desses elementos no serviço de saúde e na comunidade. Um usuário refere que:

"No nosso próprio serviço tinham pessoas que ficavam olhando com uma cara, discriminando sabe." (U17)

Alguns profissionais reconhecem a existência de situações em que a equipe parece naturalizar concepções preconceituosas a respeito da responsabilização das pessoas pela infecção do HIV:

"A equipe não tanto, mas tem também. Uma coisa assim 'olha lá o fulaninho, sempre aprontando e ó, agora viu no que deu? Agora não adianta reclamar'. Lógico que as pessoas não vão dizer isso para o paciente jamais, mas em conversinhas de corredor ainda se escuta isso." (P10)

Essa fala demonstra que a própria narrativa sobre a transmissão e a doença ensina e reproduz cadeias de compreensão baseadas nas consequências auferidas pela irresponsabilidade, indisciplina e excessos que são atribuídos ao outro considerado inconsequente. Esse processo de tentar identificar culpados pelo diagnóstico de HIV positivo parece contribuir para a manutenção do estigma.

Observa-se que a organização do trabalho das unidades básicas pode ser um facilitador para a disseminação de informações sigilosas, trazendo consequências éticas relacionadas à privacidade, ao valor e à autonomia dos usuários. Com isso, a comunicação entre a equipe sobre o atendimento e as situações vivenciais de usuários pode servir de gatilho para consolidar práticas discriminatórias. Por outro lado, os profissionais relatam que tratam o HIV com a mesma atenção com que tratam outras demandas do serviço:

"Nas unidades de saúde, para nós, um paciente com HIV é com se fosse um paciente com diabetes, a gente trata da mesma maneira. Em termos de preconceito, eu vejo assim. Têm tantos que já fazem parte do dia a dia, para nós é mais um." (P7)

Nesse sentido, não se dá visibilidade às pessoas com HIV ao manifestar que é uma doença como qualquer outra, o que reflete na abstenção de compreensão e atendimento das especificidades de cada pessoa. Ao homogeneizar as singularidades, tende-se a automatizar $\mathrm{o}$ atendimento, sem considerar a necessidade de dialogar sobre práticas sexuais e riscos de exposição individuais. Além disso, essa mecanização pode servir de proteção do confronto do profissional com suas crenças pessoais, mascarando concepções estigmatizantes.

A temática da AIDS vincula-se à necessidade de falar sobre sexo, pois trata-se de uma condição de saúde que envolve questões biomédicas, sociais, políticas e culturais. A agenda, na descentralização, quando focada somente na testagem e na medicalização, pode esquecer de fatores importantes de intervenção, que são a empatia e a importância do afeto na construção das relações dentro e entre instituições para o enfrentamento e desconstrução do estigma ${ }^{(38)}$.

A literatura aponta que a experiência de vivenciar situações de preconceito não se restringe a espaços específicos, pois sua institucionalização está presente em diferentes espaços e afeta o processo de saúde-doença( ${ }^{(23)}$. Assim, o estigma associado com estereótipos contribui para os agravos à saúde, além de comprometer o exercício da cidadania e o direito à saúde ${ }^{(17,18)}$.

Nesse sentido, um usuário relata uma experiência no serviço em que o profissional da $A B$ utiliza uma avaliação estigmatizada para referir-se ao grau de risco de exposição em uma situação descrita:

"Eu tive relação com uma guria de uma festa, e nem imaginava quem era. Ela (profissional de saúde) perguntou como era a guria, se aparentava ser drogada, uma coisa assim. Eu falei que não. Ela falou que a possibilidade não era tão grande de eu ter contraído HIV." (U14)

Ao recorrer a um perfil estereotipado de risco, a profissional perde a oportunidade de explorar diferentes situações de práticas sexuais em que o usuário poderia refletir e avaliar quais fatores envolvem a sua exposição à infecção. Ademais, possibilitaria condutas protetivas de forma a estimular a autonomia do usuário.

Sendo assim, apesar dos profissionais falarem que nos serviços há pouco estigma por parte da equipe, o fato de usarem concepções preconceituosas pode manter estigmas que resultam em relações discriminatórias. Não estimular que o usuário narre suas práticas e apresente suas dúvidas durante o atendimento obtura a oportunidade de refletir sobre ferramentas preventivas e de promoção da saúde. 
O conhecimento e enfrentamento do HIVIAIDS deve ser desenvolvido em todos os âmbitos sociais, inclusive nos serviços de saúde, de forma aberta e livre de julgamentos morais. Assim, a educação continuada pode servir como instrumento para aprimorar o aconselhamento no teste rápido na $A B$, de forma que fomente a criação de recursos de autocuidado pelos usuários, estimulando sua autonomia na gestão de sua qualidade de vida ${ }^{(39)}$.

Gestores, profissionais da saúde e demais lideranças sociais precisam assimilar que a prevenção é um direito humano e que diz respeito a todos, independentemente da identificação, concordância ou não com suas crenças, e com seus comportamentos sexuais ${ }^{(40)}$. Assim, aproveitar a expertise desenvolvida nos CTA, por exemplo, pode ser uma forma de intervenção a partir do compartilhamento das suas melhores práticas com a $A B$, por meio do apoio matricial com participação de gestores e profissionais.

Além disso, um estudo identificou que o teste rápido e o aconselhamento não estavam sendo feitos na $A B$ por livre demanda, sendo que as unidades estavam recorrendo ao agendamento ${ }^{(13)}$. Assim, sugere-se o atendimento por livre demanda, no sentido de acolher o usuário no momento de angústia e coragem de buscar a testagem. A sala de espera também pode ser usada como espaço para orientação sobre sexualidade e prevenção de Infecções Sexualmente Transmissíveis (IST).

Mesmo que os profissionais participantes deste estudo tenham realizado o curso de testagem para HIVIAIDS, ainda demonstraram discriminação e preconceito diante das questões da pesquisa. Assim, sugere-se também que realizem cursos que possam trabalhar sua sexualidade, para que tenham condições de auxiliar nas questões sobre a sexualidade de sua clientela. Ressalta-se, ainda, que a sensibilização dos profissionais de saúde quanto aos problemas enfrentados pela clientela sobre o HIVIAIDS é fundamental para a promoção à saúde desses indivíduos, dando oportunidade de escuta ativa, disponibilidade de insumos de proteção à transmissão do vírus e informações quanto às boas práticas sexuais, garantindo a vivência satisfatória de sua saúde sexual( ${ }^{(41)}$.

Ações nesse sentido têm como impacto a abertura ao diálogo, aumento na busca do serviço e consolidação de estratégias de prevenção, com vistas ao controle da epidemia. Para avaliar os resultados dessa intervenção, é possível implementar um estudo piloto com duas unidades para monitorar o desenvolvimento dessas práticas. Posteriormente, realizar estudo comparativo para averiguar a presença do estigma entre os serviços nos quais foram aplicadas e aqueles onde não foram aplicadas as intervenções.

Como limitação do estudo, vale ressaltar que todos os entrevistados consideravam ter o diagnóstico negativo de HIV, o que pode ter influenciado nas respostas. Devido ao número de participantes e serviços, os dados não podem ser generalizados, porém, os resultados refletem a complexidade do estigma relacionado ao HIVIAIDS dentro do contexto da $A B$.

\section{CONSIDERAÇÕES FINAIS}

A partir do objetivo proposto, compreende-se que o estigma associado ao HIV se faz presente nas diferentes falas dos entrevistados. De forma geral, profissionais e usuários percebem estigma e discriminação na comunidade e nos serviços de saúde, sem uma implicação crítica sobre sua participação nesse processo.

Diante da descentralização do teste rápido de HIV e também de outras Infecções Sexualmente Transmissíveis (IST), o desafio da AB é não ser estimuladora de estigmas. É preciso reconhecer que a AIDS tem suas especificidades sócio-históricas que se desdobraram na constituição de estigmas e relações discriminatórias. Dessa forma, tornase relevante compreender os conceitos que embasam a identificação de cada população como vulnerável ao HIV/ AIDS para que os serviços de saúde produzam ações inclusivas de promoção de saúde.

\section{AGRADECIMENTOS E CONFLITOS DE INTERESSE}

Agradecimento ao Ministério da Ciência, Tecnologia e Inovação e à Fundação de Amparo à Pesquisa do Estado do Rio Grande do Sul.

O manuscrito não apresenta relações que possam implicar em potencial conflitos de interesse.

\section{CONTRIBUIÇÕES}

No presente estudo, todos os autores participaram igualmente na construção do artigo, análise dos dados, estruturação dos resultados e execução do manuscrito. 


\section{REFERÊNCIAS}

1. UNAIDS. Global AIDS Update. Aids [Internet]. 2016 [acesso em 2017 Mar 10];17 Suppl 4:13. Disponível em: http://pesquisa.bvsalud.org/portal/resource/pt/mdl-15080170

2. Ministério da Saúde (BR). Boletim Epidemiológico Hiv/Aids da Secretaria de Vigilância em Saúde - Ministério da Saúde. Bol Epidemiol [Internet]. 2017 [acesso em 2017 Fev 16];48(1):52. Disponível em: http://portalsaude. saude.gov.br/images/pdf/2017/janeiro/05/2016_034-Aids_publicacao.pdf

3. Goffman E. Estigma: la identidad deteriorada. 5a ed. Buenos Aires: Amorrortu Editores; 1993.

4. Parker R, Aggleton P. HIV and AIDS-related stigma and discrimination: a conceptual framework and implications for action. Soc Sci Med. 2003;57(1):13-24.

5. Bos AER, Pryor JB, Reeder GD, Stutterheim SE. Stigma: advances in theory and research. Basic Appl Soc Psych. 2013;35(1):1-9.

6. Monteiro SS, Villela WV, Soares PS. The interaction between axes of inequality in studies on discrimination, stigma and HIVIAIDS: contributions to the recent international literature. Glob Public Health. 2013;8(5):519-33.

7. Phelan JC, Link BG, Dovidio JF. Stigma and prejudice: one animal or two? Soc Sci Med. 2008;67(3):358-67.

8. Andrinopoulos K, Clum G, Murphy D, Harper G, Perez L, Xu J, et al. Health related quality of life and psychosocial correlates among HIV-infected adolescent and young adult women in the US. AIDS Educ Prev [Internet]. 2011 [acesso em 2017 Ago 27];23(4):367-81. Disponível em: http://www.scopus.com/inward/record.url?eid=2-s2.080052047231\&partnerID=40\&md5=c05d984a74701bc6abe9147f7f74f78c

9. Costa AB, Bandeira DR, Nardi HC. Systematic review of instruments measuring homophobia and related constructs. J Appl Soc Psychol. 2013;43(6):1324-32.

10. Garcia J, Parker C, Parker RG, Wilson PA, Philbin M, Hirsch JS. Psychosocial implications of homophobia and hiv stigma in social support networks: insights for high-impact HIV prevention among black men who have sex with men. Health Educ Behav. 2016;43(2):217-25.

11. Ministério da Saúde (BR). Oficina de aconselhamento em DST/HIVIAIDS para a atenção básica. Brasília: Ministério da Saúde; 2005.

12. Brasil. Portaria $n^{\circ} 77$, de 12 de janeiro de 2012. Dispõe sobre a realização de testes rápidos, na atenção básica, para a detecção de HIV e sífilis [Internet]. Brasília: Diário Oficial da República Federativa do Brasil; 2012. Disponível em: http://bvsms.saude.gov.br/bvs/saudelegis/gm/2012/prt0077_12_01_2012.html

13. Rocha KB, Ew RAS, Moro LM, Zanardo GLP, Pizzinato A. Aconselhamento na perspectiva de profissionais da atenção básica : desafios na descentralização do teste rápido HIV / Aids. Ciênc Psicol. 2018;12(1):67-78.

14. Ministério da Saúde (BR). Política Nacional de Promoção da Saúde. Brasília: Ministério da Saúde; 2010. v. 8.

15. Zambenedetti G, Both NS. "A via que facilita é a mesma que dificulta": estigma e atenção em HIV-Aids na estratégia saúde da família - ESF. Fractal Rev Psicol. 2013;25(1):41-58.

16. Zambenedetti G, Silva RAN. Descentralização da atenção em HIV-Aids: Para a atenção básica: tensões e potencialidades. Physis (Rio de J). 2016;26(3):785-806.

17. Parker R. Interseções entre estigma, preconceito e discriminação na saúde pública mundial. In: Monteiro $S$, Villela WV. Estigma e saúde. Rio de Janeiro: Fiocruz; 2013. p. 25-46.

18. Major B, Dovidio JF, Link BG, Calabrese SK. Stigma and its implication for health: introduction and overview. In: Major B, Dovidio JF, Link BG. The Oxford handbook of stigma, discrimination, and health. Nova York: Oxford University Press; 2017. p. 3-28.

19. Flick U. An introduction to qualitative research. $5^{\text {th }}$ ed. Londres: SAGE; 2014.

20. Braun V, Clarke V. Using thematic analysis in psychology. Qual Res Psychol [Internet]. 2006 [acesso em 2017 Out 13];3(2):77-101. Disponível em: http://eprints.uwe.ac.uk/11735

21. Greco DB. Trinta anos de enfrentamento à epidemia da Aids no Brasil, 1985-2015. Ciên Saúde Colet [Internet]. 2016 [acesso em 2017 Nov 8];21(5):1553-64. Disponível em: http://www.scielo.br/scielo.php?script=sci_ arttext\&pid=S1413-81232016000501553\&lng=pt\&tIng=pt 
22. Villela WV, Monteiro S. Gênero, estigma e saúde: reflexões a partir da prostituição, do aborto e do HIV/aids entre mulheres. Epidemiol Serv Saúde [Internet]. 2015 [acesso em 2017 Nov 23];24(3):531-40. Disponível em: http://www.iec.pa.gov.br/template_doi_ess.php?doi=10.5123/S1679-49742015000300019\&scielo $=$ S2237-96222015000300531

23. Venturi G. Misoginia, homofobia, racismo e "gerontofobia": contribuições de análises da opinião pública para a prevenção. In: Paiva VSF, Ayres JR, Buchalla CM. Vulnerabilidade e direitos humanos - prevenção e promoção da saúde: Livro 1. Curitiba: Juruá; 2012. p. 95-110.

24. Cabral JVB, Oliveira FHPC, Messias DCA, Santos KLLM, Bastos V. A percepção de vulnerabilidade da população adolescente sobre o HIVIAIDS. Espaç Saúde. 2016;17(2):212-9.

25. Secretaria de Estado da Saúde do Rio Grande do Sul. Boletim Epidemiológico: HIVIAIDS - RS. Porto Alegre: Escola de Saúde Pública; 2017.

26. Panarra BACS, Teixeira E, Palmeira IP, Rodrigues ILA, Ferreira AMR. Vítimas e culpadas: representações sociais sobre mulheres que vivem com HIV. Rev Cuid (Bucaramanga) [Internet]. 2017 [acesso em 2018 Jan 7];8(3):1887-98. Disponível em: https://www.revistacuidarte.org/index.php/cuidarte/article/view/451

27. Carneiro AJS, Coelho EDAC. Aconselhamento na testagem anti-HIV no ciclo gravídico-puerperal: o olhar da integralidade. Ciênc Saúde Colet. 2010;15(Supl 1):1216-26.

28. Zucchi EM, Paiva VSF, França I Júnior. Intervenções para reduzir o estigma da AIDS no Brasil: uma revisão crítica. Temas Psicol [Internet]. 2013 [acesso em 2017 Dez 19];21(3):1067-87. Disponível em: http://pepsic. bvsalud.org/pdf/tp/v21n3/v21n3a17.pdf

29. Bittencourt GKGD, Moreira MASP, Meira LCS, Nóbrega MML, Nogueira JA, Silva AO. Beliefs of older adults about their vulnerability to HIV/Aids, for the construction of nursing diagnoses. Rev Bras Enferm [Internet]. 2015 [acesso em 2017 Nov 23];68(4):579-85. Disponível em: http://www.scielo.br/scielo.php?script=sci_arttext\&pid=S003471672015000400579\&lng=en\&nrm=iso\&tlng=pt

30. Madi CR, Gomes JL, Louzada TG. Revisão bibliográfica da publicação "Mais 60 - Estudos sobre envelhecimento" Estud Envelhec. 2017;28(67):56-83.

31. Aguiar RA, Ciosak SI. Late diagnosis and vulnerabilities of the elderly living with HIVIAIDS. Rev Esc Enferm USP [Internet]. 2015 [acesso em 2018 Jan 7];49(2):229-35. Disponível em: http://www.scielo.br/scielo.php?script=sci_ar ttext\&pid=S0080-62342015000200229

32. Andrade J, Ayres JA, Alencar RA, Duarte MTC, Parada CMGL. Vulnerabilidade de idosos a infecções sexualmente transmissíveis. Acta Paul Enferm [Internet]. 2017 [acesso em 2017 Set 19];30(1):8-15. Disponível em: http:// www.scielo.br/scielo.php?script=sci_arttext\&pid=S0103-21002017000100008\&lng=pt\&tlng=pt

33. Damaceno AN, Bandeira D, Hodali N, Weiller TH. Acesso de primeiro contato na Atenção Primária à Saúde: Revisão integrativa. Rev APS [Internet]. 2016 [acesso em 2017 Dez 19];19(1):122-38. Disponível em: https:// aps.ufjf.emnuvens.com.br/aps/article/view/2521/944

34. Zambenedetti G, Silva RAN. O paradoxo do território e os processos de estigmatização no acesso ao diagnóstico de HIV na atenção básica em saúde. Estud Psicol [Internet]. 2015 [acesso em 2018 Jan 10];20(4):229-40. Disponível em: http://www.gnresearch.org/doi/10.5935/1678-4669.20150024

35. Lopes AS, Vilar RLA, Melo RHV, França RCDS. O acolhimento na Atenção Básica em saúde: relações de reciprocidade entre trabalhadores e usuários. Saúde Debate [Internet]. 2015 [acesso em 2017 Nov 14];39(104):11423. Disponível em: http://www.scielo.br/scielo.php?script=sci_arttext\&pid=S0103-11042015000100114\&lng=pt \&tlng=pt

36. Mak WWS, Cheng SSY, Law RW, Cheng WWL, Chan F. Reducing HIV-related stigma among health-care professionals: A game-based experiential approach. AIDS Care. 2015;27(7):855-9.

37. Antunes L, Camargo BV, Bousfield ABS. Representações sociais e estereótipos sobre aids e pessoas que vivem com HIV/Aids. Psicol Teor Prát [Internet]. 2014 [acesso em 2018 Jan 10];16(3):43-57. Disponível em: http:// pepsic.bvsalud.org/scielo.php?script=sci_arttext\&pid=S1516-36872014000300004\&lng=pt\&nrm=iso\&tlng=pt

38. Parker R. A cura da AIDS. 2014 [acesso em 2017 Dez 5]. Disponível em: http://abiaids.org.br/?p=27249 
39. Sobral ILL, Machado LDS, Gomes SHP, Pequeno AMC, Nuto SAS, Machado MFAS. Conhecimento de profissionais da Atenção Básica sobre competência de promoção da saúde. Rev Bras Promoç Saúde. 2018;31(2):1-7.

40. Paiva VSF. Psicologia na saúde: sociopsicológica ou psicossocial? Inovações do campo no contexto da resposta brasileira à aids. Temas Psicol [Internet]. 2013 [acesso em 2018 Jan 11];21(3):531-49. Disponível em: http:// pepsic.bvsalud.org/pdf/tp/v21n3/v21n3a02.pdf

41. Diógenes MAR, Portela IB, Sá RC, Valente MMQ. Sexualidade de portadores do vírus da imunodeficiência humana em face à doença: Revisão integrativa. Rev Bras Promoç Saúde. 2017;27(4):550-9.

\section{Endereço do primeiro autor:}

Raquel de Andrade Souza Ew

Pontifícia Universidade Católica do Rio Grande do Sul

Av. Ipiranga, 6681 / Prédio 11 / sala 931

Bairro: Partenon

CEP: 90619-900 - Porto Alegre - RS - Brasil

E-mail: raquelew@hotmail.com

\section{Endereço para correspondência:}

Kátia Bones Rocha

Pontifícia Universidade Católica do Rio Grande do Sul

Av. Ipiranga, 6681 / Prédio 11 / sala 931

Bairro: Partenon

CEP: 90619-900 - Porto Alegre - RS - Brasil

E-mail: katia.rocha@pucrs.br 